\title{
Evidence for a Second Transport Porosity for the Diffusion of Tritiated Water (HTO) in a Sedimentary Rock (Opalinus Clay - OPA): Application of Through- and Out-Diffusion Techniques
}

\author{
L. R. VAN LOON* and A. JAKOB \\ Laboratory for Waste Management, Paul Scherrer Institut, CH-5232 Villigen, PSI, \\ Switzerland
}

(Received: 16 July 2004; accepted in final form: 9 December 2004)

\begin{abstract}
The diffusion of tritiated water (HTO) in Opalinus clay (OPA) samples from bore cores from the Benken area (Northern Switzerland) was studied using the radial through- and out-diffusion technique. Results from inverse modelling of out-diffusion data for HTO indicated the presence of two preferential diffusion pathways: a fast and a slow one. Analysing through-diffusion data, however, provides hardly any information concerning a second transport-relevant porosity. Only by also analysing the out-diffusion phase can finer details of the diffusion process and information on sample heterogeneity be recognised. The extracted values for the effective diffusion coefficient are in the order of $3 \times 10^{-11} \mathrm{~m}^{2} \mathrm{~s}^{-1}$ for the faster transport porosity and roughly an order of magnitude smaller for the slower type of porosity. We had to account for tritium sorption on the clay minerals by a small but non-zero $K_{\mathrm{d}}$-value in the order of $10^{-5} \mathrm{~m}^{3} \mathrm{~kg}^{-1}$ in order to reproduce the data with acceptable precision. In the model applied both porosities are considered as being independent from each other. Roughly $30 \%$ of the tracer diffused through the second, slower porosity; such a fact might be interesting for future performance assessments for radioactive waste repositories hosted by clay formations. Based on our present picture from water-saturated OPA, on a microscopic scale three different kind of waters can be discriminated: free water, double layer water and interlayer water. However, using HTO as tracer only, it could not be deduced which type of water-filled pores finally account for the transport-relevant porosity.
\end{abstract}

Key words: through-diffusion, out-diffusion, tritiated water, experiment, inverse modelling, Opalinus clay. ${ }^{*}$ Author for correspondence: Tel.: +41-56-3102257; Fax: +41-56-3104438; E-mail:
luc.vanloon@psi.ch 


\section{List of Symbols}

\begin{tabular}{|c|c|c|}
\hline Symbol & Unit & Meaning \\
\hline$A_{\text {dif }}$ & $\mathrm{Bq}$ & tracer activity diffused through the sample \\
\hline$C$ & $\mathrm{~Bq} \mathrm{~m}^{-3}$ & amount of tracer per unit volume of liquid phase \\
\hline$C_{0}$ & $\mathrm{~Bq} \mathrm{~m}^{-3}$ & averaged tracer concentration in the reservoir for $0 \leq r<r_{\text {int }}$ \\
\hline$D_{a}$ & $\mathrm{~m}^{2} \mathrm{~s}^{-1}$ & 1D apparent diffusion coefficient $\left(D_{\mathrm{a}}=D_{\mathrm{e}} / \alpha\right)$ \\
\hline$D_{e}$ & $\mathrm{~m}^{2} \mathrm{~s}^{-1}$ & $1 \mathrm{D}$ effective diffusion coefficient $\left(D_{\mathrm{e}}=\varepsilon D_{\mathrm{p}}\right)$ \\
\hline$D_{p}$ & $\mathrm{~m}^{2} \mathrm{~s}^{-1}$ & 1D pore diffusion coefficient \\
\hline$f$ & $\operatorname{cps~Bq}{ }^{-1}$ & counting efficiency (cps ... counts per seconds) \\
\hline$h$ & $\mathrm{~m}$ & height of the cylindrical Opalinus clay sample \\
\hline$j_{\mathrm{ext}}$ & $\mathrm{Bq} \mathrm{m}^{-2} \mathrm{~s}^{-1}$ & computed tracer flux across the outer boundary \\
\hline$j_{i}$ & $\mathrm{~Bq} \mathrm{~m}^{-2} \mathrm{~s}^{-1}$ & measured tracer flux across the outer boundary at time $t_{i}$ \\
\hline$J_{0}$ & - & Bessel function of the first kind and order zero \\
\hline$K_{\mathrm{d}}$ & $\mathrm{m}^{3} \mathrm{~kg}^{-1}$ & Linear sorption distribution coefficient \\
\hline$L$ & $\mathrm{~m}^{2}$ & Geometry factor (see Equation 15b) \\
\hline$m$ & $\mathrm{~Bq}$ & total diffused tracer mass (activity) \\
\hline$N_{\text {dif }}$ & cpm & total amount of tracer diffused (in counts per minute) \\
\hline$N_{s}$ & cpm & net count rate in counts per minute \\
\hline$N_{T}$ & cpm & counting rate in counts per minute \\
\hline$N_{0}$ & cpm & background count rate in counts per minute \\
\hline$r$ & $\mathrm{~m}$ & cylindrical co-ordinate \\
\hline$r_{\text {int }}$ & $\mathrm{m}$ & inner radius of the hollow cylindrical sample \\
\hline$r_{\text {ext }}$ & $\mathrm{m}$ & outer radius of the hollow cylindrical sample \\
\hline$R_{\mathrm{d}}$ & $\mathrm{m}^{3} \mathrm{~kg}^{-1}$ & tracer distribution ratio $\left(R_{\mathrm{d}}=S / C\right)$ \\
\hline$S$ & $\mathrm{~Bq} \mathrm{~kg}^{-1}$ & amount of absorbed tracer per unit mass of solid phase \\
\hline$S_{\text {ext }}$ & $\mathrm{m}^{2}$ & cylindrical surface accessible for diffusion at the outer \\
\hline$t$ & s & radiustime \\
\hline$T$ & s & counting time \\
\hline$V_{\text {dead }}$ & $\mathrm{m}^{3}$ & dead volume; i.e. volume of the tubings, grooves, etc. \\
\hline$V_{\text {high }}$ & $\mathrm{m}^{3}$ & volume of the solution in the high-concentration reservoir \\
\hline$V_{\text {low }}$ & $\mathrm{m}^{3}$ & volume of the solution in the low-concentration reservoir \\
\hline$V_{\text {sample }}$ & $\mathrm{m}^{3}$ & sampling volume for the activity measurement \\
\hline$Y_{0}$ & - & Bessel function of the second kind and order zero \\
\hline \multicolumn{3}{|c|}{ Greek symbols } \\
\hline$\alpha$ & - & rock capacity factor \\
\hline$\chi^{2}$ & - & chi-square merit function \\
\hline$\partial$ & - & partial derivative \\
\hline$\Delta$ & - & increment in the quantity under consideration \\
\hline$\varepsilon$ & - & transport (diffusion accessible) porosity of the sample \\
\hline$\pi$ & - & $3.1415926535 \ldots$ \\
\hline$\rho$ & $\mathrm{kg} \mathrm{m}^{-3}$ & density of the solid phase \\
\hline
\end{tabular}




\section{Introduction}

Several European countries consider argillaceous formations as potential host rocks for radioactive waste disposal (Andra, 2001; Ondraf, 2001; Nagra, 2002a). The main reasons for this are (1) the very low permeability of such formations and (2) the pronounced capability for an efficient retardation of mobile radionuclides, which make them ideal containment for isolating radioactive contaminants for very long time periods. Due to the absence of fractures and due to the low permeability of the clay, the main process responsible for transport of radionuclides in such formations is molecular diffusion. It is therefore very important to understand the diffusion behaviour of radionuclides in these argillaceous materials on a fundamental level.

Sedimentary rocks are composed of different types of clay minerals, calcite, quartz, feldspar etc; resulting in a complicated 3D-network of pores. Diffusion studies on such materials, however, always involve treatment as a homogeneous single porosity medium, characterised by a 1Deffective diffusion coefficient $D_{\mathrm{e}}$ and a rock capacity factor $\alpha$ (for example, see the papers by Van Loon et al. (2003a,b), Put and De Canniere (1994) Henrion et al. (1991) and Bourke et al. (1993)). From a structural point of view, however, a much more complicated picture is expected. This apparent contradiction between observations on a microscopic scale on clay and modelling of tracer through-diffusion (or in-diffusion) in the frame of a homogeneous and isotropic 1D through-diffusion/sorption model thus indicates that generally one type of porosity dominates diffusive transport in such materials. Moreover, as we will demonstrate, the different diffusion techniques often used (through-diffusion, in-diffusion, leaching, etc.) are not suited for resolving the finer details of the diffusion processes in such complex materials.

Our investigations focus on a radial through- and out-diffusion technique for studying the diffusion behaviour of HTO in a compacted argillaceous rock, Opalinus clay (OPA), containing mainly kaolinite, illite and illite/smectite clay minerals. The radial through-diffusion technique has been published elsewhere (Van Loon et al., 2004). The radial out-diffusion technique, however, is new and allows obtaining a more detailed picture of the diffusion processes occurring in the rock.

\section{Materials and Methods}

\subsection{CLAY ROCK SAMPLES}

We used an OPA sample taken from a depth of $-630 \mathrm{~m}$ at Benken (BE), a location situated in the North-Eastern part of Switzerland. OPA is currently under investigation as a potential host rock for a high-level 
radioactive waste repository in Switzerland (Nagra, 2002a). The OPA was deposited about 180 million years ago as a marine sediment consisting of fine mud particles. It has a thickness of $\sim 100 \mathrm{~m}$ and contains between $40 \%$ and $80 \%$ clay minerals, $10 \%$ of which are capable of swelling (Nagra, 2001, 2002a); the averaged mineral composition of the rock is given in Table I. The clay platelets are orientated preferentially in a direction parallel to the surface (Nagra, 2002a, pp. 222-224). Such a preferential orientation results in anisotropic diffusion processes in the OPA (Van Loon et al., 2004). For our diffusion experiments cylindrical samples with a central cavity were prepared by lathing, as described in Van Loon et al. (2004); the dimensions of the samples and some other important data of the experiments are compiled in Table II.

\subsection{PORE WATER}

The composition of the artificial pore-water assumed to be in equilibrium with the OPA is given in Table III (Pearson, 2000).

\subsection{RADIAL DIFFUSION TECHNIQUE}

The details of the diffusion cell and the experimental technique used are described in Van Loon et al. (2004). The diffusion cell was loaded with the hollow cylindrical OPA sample and a confining pressure of $15 \mathrm{MPa}$, simulating the in situ overburden at a depth of roughly $630 \mathrm{~m}$,

Table I. Average mineral composition of Benken OPA (Nagra, 2001)

\begin{tabular}{lc}
\hline Mineral & Weight $\%$ \\
\hline Calcite & $16 \pm 10$ \\
Dolomite/ankerite & $1.0 \pm 0.4$ \\
Siderite & $4.0 \pm 2.4$ \\
Quartz & $20 \pm 1$ \\
K-Feldspar & $2 \pm 1$ \\
Albite & $1.0 \pm 0.3$ \\
Pyrite & $1.1 \pm 1$ \\
& \\
Clay minerals & \\
Illite & $18 \pm 6$ \\
Illite/smectite mixed layers & $14 \pm 4$ \\
Chlorite & $5 \pm 2$ \\
Kaolinite & $17 \pm 6$ \\
\hline
\end{tabular}


Table II. Dimension of the OPA sample and predefined input parameter values together with their $1 \sigma$-errors needed for the numerical calculations

\begin{tabular}{lllr}
\hline Parameter & Unit & Meaning & Value \\
\hline$r_{\text {int }} \times 10^{3}$ & $\mathrm{~m}$ & Inner radius of the cylindrical OPA sample & $6.58 \pm 0.02$ \\
$r_{\text {ext }} \times 10^{3}$ & $\mathrm{~m}$ & Outer radius of the cylindrical OPA sample & $25.25 \pm 0.02$ \\
$h \times 10^{3}$ & $\mathrm{~m}$ & Height of the OPA sample & $51.98 \pm 0.02$ \\
$V_{\text {high }} \times 10^{6}$ & $\mathrm{~m}^{3}$ & Volume of the traced solution in the reservoir & $202.0 \pm 0.1$ \\
$V_{\text {low }} \times 10^{6}$ & $\mathrm{~m}^{3}$ & Volume of the low concentration solution & $20.0 \pm 0.1$ \\
$\rho$ & $\mathrm{kg} \mathrm{m}^{-3}$ & Solid phase density & $2716 \pm 30$ \\
$C_{0} \times 10^{-9}$ & $\mathrm{~Bq} \mathrm{~m}^{-3}$ & Weighted average reservoir concentration & $1.14 \pm 0.04$ \\
\hline
\end{tabular}

Table III. Composition of the synthetic OPA pore water used in the diffusion experiments (Pearson et al. 2000; Van Loon et al., 2003b). The relative error on the concentration is $\max .0 .5 \%$

\begin{tabular}{ll}
\hline Element & mol.dm \\
\hline $\mathrm{Na}$ & $1.50 \times 10^{-1}$ \\
$\mathrm{~K}$ & $4.31 \times 10^{-3}$ \\
$\mathrm{Mg}$ & $5.21 \times 10^{-3}$ \\
$\mathrm{Ca}$ & $7.24 \times 10^{-3}$ \\
$\mathrm{Sr}$ & $3.97 \times 10^{-4}$ \\
$\mathrm{Cl}$ & $1.60 \times 10^{-1}$ \\
$\mathrm{SO}_{4}$ & $1.00 \times 10^{-2}$ \\
$\mathrm{CO}_{3} / \mathrm{HCO}_{3}$ & $3.11 \times 10^{-4}$ \\
$\mathrm{pH}$ & 7.9 \\
$\Sigma$ cations & $1.80 \times 10^{-1} \mathrm{eq} \mathrm{dm}^{-3}$ \\
$\Sigma$ anions & $1.80 \times 10^{-1} \mathrm{eq} \mathrm{dm} \mathrm{dm}^{-3}$ \\
$\mathrm{I}$ & 0.20 \\
\hline
\end{tabular}

was applied to the sample. A schematic presentation of the experimental set-up is given in Figure 1. The volume of the solution with the high tracer concentration was $202 \mathrm{~cm}^{3}$ and that of the low concentration solution $20 \mathrm{~cm}^{3}$. The samples were re-hydrated using the synthetic pore water specified in Table III. After hydration, which took approximately one month time, tritiated water (HTO) was added to the solution in the highconcentration container, thus starting tracer through-diffusion. The activity of HTO at elapsed time equal to zero was $1.21 \times 10^{9} \mathrm{~Bq} \mathrm{~m}^{-3}$. (The half-life of tritium is 12.323 years, hence much larger than the duration of 


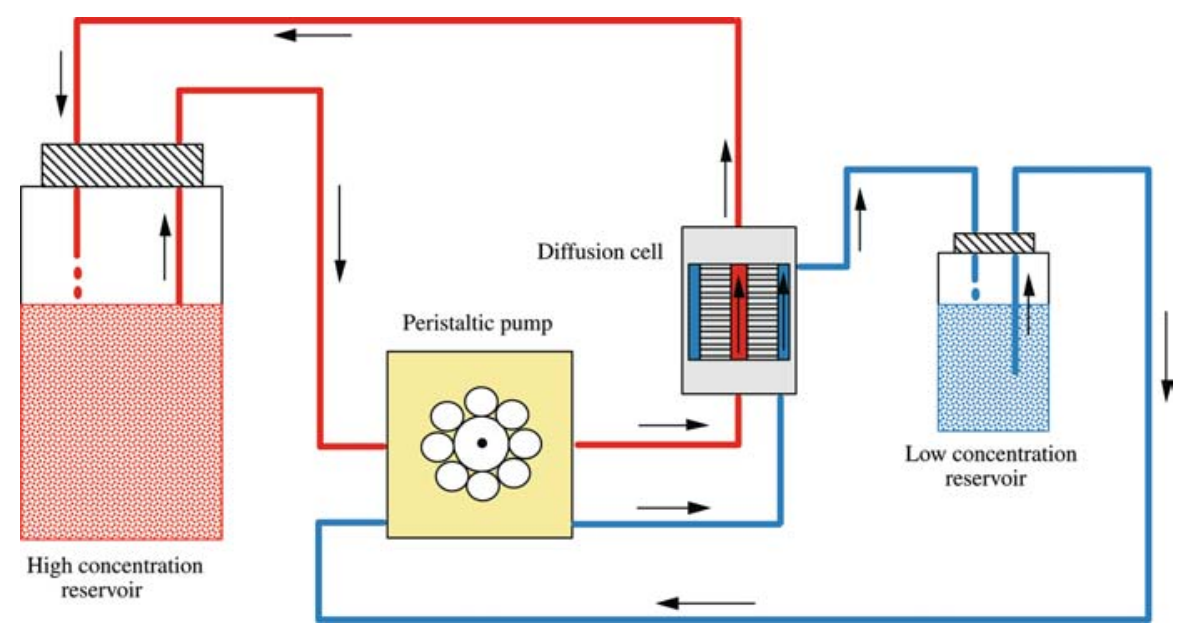

Figure 1. Schematic view of the radial diffusion set-up used in the through- and out-diffusion experiments.

our experiments which is in the order of a few months; thus as a tracer, it can be considered stable for all practical purposes. Therefore we specify the tracer concentration in units of $\mathrm{Bq} \mathrm{m}^{-3}$ without any dates.) The solutions in the small, low-concentration side containers were replaced after a given time interval, $\Delta t$, to keep the activity of the tracer in this compartment as low as possible, i.e. for practical purposes $<1 \%$ of the tracer concentration in the high-concentration compartment. The activity of HTO in solution was measured by liquid scintillation counting as described in Van Loon et al. (2003a). When the diffusion process was in a steady-state phase, i.e. constant diffusive flux across the down-stream boundary, the tracer through-diffusion phase was stopped. The activity left in the tubing was removed by flushing the system with artificial pore water for half an hour. The solutions in both containers were then replaced by artificial pore water without tracer, starting the out-diffusion phase. At selected time intervals, the activity in the solutions was measured and the solutions replaced by fresh ones. This procedure was repeated until all the activity in the sample had diffused out. Care had to be taken that no additional hydraulic head difference resulting in an additional advective tracer flux was generated. Assuming a value for the hydraulic conductivity which is (3-6) $10^{-14} \mathrm{~m} \mathrm{~s}^{-1}$ parallel to bedding (Nagra, 2002b, p. 322) and a maximum pressure difference across the diffusion cell which corresponds to a $10 \mathrm{~cm}$ water column, results in an additional advective flux which is at least two orders of magnitude smaller than the diffusive tracer flux. Hence, such an additional driving force for tracer transport could be neglected. 


\subsection{DATA PROCESSING FOR THROUGH-DIFFUSION}

After a time $t_{i}$, the tracer solution in the low activity container, having a volume $V_{\text {low }}$, was replaced by the same volume of tracer-free artificial pore water. A given volume $V_{\text {sample }}$ of this sampled solution was used for the activity measurements at $t=t_{i}$ (counting time is $T$ ), yielding a count rate, $N_{T}^{t_{i}}$ in counts per minute (cpm). The background was measured under similar conditions resulting in a count rate $N_{0}^{t_{i}}(\mathrm{cpm})$. From these two measurements, a net count rate, $N_{s}^{t_{i}}(\mathrm{cpm})$, was calculated according to

$$
N_{s}^{t_{i}}=N_{T}^{t_{i}}-N_{\phi}^{t_{i}} .
$$

The total amount of tracer [cpm] diffused through the sample during a time interval $\Delta t_{i}=t_{i}-t_{i-1}$ is calculated by

$$
N_{\text {dif }}^{\Delta t_{i}}=\frac{V_{\text {low }}}{V_{\text {sample }}} N_{s}^{t_{i}}+\frac{V_{\text {dead }}}{V_{\text {sample }}}\left(N_{s}^{t_{i}}-N_{s}^{t_{i-1}}\right),
$$

where $V_{\text {dead }}$ is the volume of the traced solution that is not replaced when changing the solution in the low concentration reservoir and represents the dead volume of the experiment, i.e. the volume of the tubing, the grooves in the sample holder wall and the filter pore space. The negative term in Equation (2) represents the correction for the activity left in this dead volume when replacing the solution in the container. At time $t=t_{0}=0$ the count rate $N_{s}^{t_{0}}$ is zero. Hence, for $i=1$, i.e. the first sampling, the total amount of tracer (cpm) diffused is

$$
N_{\text {dif }}^{\Delta t_{1}}=\frac{V_{\text {low }}+V_{\text {dead }}}{V_{\text {sample }}} N_{s}^{t_{1}} .
$$

The tracer activity diffused $A_{\text {dif }}(\mathrm{Bq})$ through the rock during a time $\Delta t_{i}$ is now given by

$$
A_{\mathrm{dif}}^{\Delta t_{i}}=\frac{N_{\mathrm{dif}}^{\Delta t_{i}}}{60 \cdot f}
$$

where $f\left(\mathrm{cps} \mathrm{Bq}^{-1}\right)$ is the counting efficiency $\left(f=0.35 \mathrm{cps} \mathrm{Bq}^{-1}\right.$ for HTO). The diffusive tracer flux $j_{r \text {,ext }}^{t_{i}}\left(\mathrm{~Bq} \mathrm{~m}^{-2} \mathrm{~s}^{-1}\right)$, averaged over the time interval $\Delta t_{i}$ is

$$
j_{i}\left(r_{\mathrm{ext}}, \frac{t_{i}+t_{i-1}}{2}\right)=\frac{A_{\mathrm{dif}}^{\Delta t_{i}}}{S_{\mathrm{ext}} \cdot \Delta t_{\mathrm{i}}},
$$


where $S_{\text {ext }}$ represents the cylindrical area $\left(\mathrm{m}^{2}\right)$ accessible for tracer through diffusion at the outer boundary at $r=r_{\text {ext }}$ of the sample. The accumulated activity diffused through the rock sample after diffusion time $t_{n}$ is

$$
A_{\text {dif }}\left(r_{\text {ext }}, t_{n}\right)=\sum_{i=1}^{n} A_{\text {dif }}^{\Delta t_{i}} .
$$

(Note: the flux data are allocated to the middle of the time intervals. However, total diffused mass is allocated to the end of the sampling period.) The uncertainty on the flux and the total diffused activity was calculated as described in Van Loon and Soler (2004).

\subsection{DATA PROCESSING FOR OUT-DIFFUSION}

After reaching the steady-state condition for tracer through-diffusion, the solutions in both containers were replaced by the same volume of tracerfree artificial porewater thereby starting tracer out-diffusion across both boundaries. As described above for tracer through-diffusion, periodically the traced solutions were replaced for the activity measurements until all of the tracer had diffused out of the sample. The sampling procedure and the subsequent data processing remained the same as that of the former through-diffusion phase.

\section{The Formalism for Through- and Out-Diffusion in Case of a Hollow Cylinder}

The migration of radionuclides through a hollow cylindrical OPA sample saturated with artificial pore water is assumed to be dominated only by molecular diffusion due to the low permeability and the absence of fractures. Radionuclides are transferred by molecular diffusion through interstitial water from the central cavity to the outer surface of the sample and then released to the water on the reservoir side. For the modelling and for simplicity it is assumed that the OPA sample is homogeneous and isotropic with regard to its transport properties and that the tracer concentration gradient across the sample is homogeneous as well. Therefore, tracer through-diffusion may be well described by a one-dimensional single porosity medium model; and the diffusion coefficient is assumed to be constant in space and time and furthermore - for simplicity's sake - independent of the tracer concentration. Based on these assumptions it is concluded that the flux of a tracer through a porous medium is dependent on the following mechanisms:

- molecular diffusion in the aqueous phase and

- possible sorption processes on the solid phase surfaces, 
and on geometrical aspects such as

- the pore constrictivity and

- the tortuosity of the OPA sample.

In order to derive an adequate description of the diffusion/sorption problem, one has to solve the diffusion equation in cylindrical co-ordinates with appropriate initial and boundary conditions consistent to the experiments.

Based on mass-balance considerations for a representative elementary volume and for stable tracers, diffusion is expressed by Fick's second law:

$$
\frac{\partial}{\partial t}\left(C+\frac{1-\varepsilon}{\varepsilon} \rho S\right)=D_{\mathrm{p}}\left(\frac{\partial^{2} C}{\partial r^{2}}+\frac{1}{r} \frac{\partial C}{\partial r}\right) .
$$

In this equation $t(\mathrm{~s})$ is the time, $C\left(\mathrm{~Bq} / \mathrm{m}^{3}\right)$ the tracer concentration in the mobile phase, $\varepsilon(-)$ the connected porosity of the clay, $\rho\left(\mathrm{kg} / \mathrm{m}^{3}\right)$ the solid density of the diffusion barrier, $S(\mathrm{~Bq} / \mathrm{kg})$ the amount of tracer absorbed onto the solid phase per unit mass of solid phase, $D_{\mathrm{p}}\left(\mathrm{m}^{2} / \mathrm{s}\right)$ the pore diffusion coefficient, and $r(\mathrm{~m})$ the spatial co-ordinate. $D_{\mathrm{p}}$ is, in general, a second-rank tensor for diffusion in a porous medium and includes the effects of tortuosity and constrictivity. However, dealing with an isotropic porous medium the tensor is reduced to a scalar.

For possible sorption processes for simplicity $S=f(C)$ is assumed, where $f(C)=K_{\mathrm{d}} C$ denotes a linear sorption isotherm and $K_{\mathrm{d}}\left(\mathrm{m}^{3} / \mathrm{kg}\right)$ is the linear sorption equilibrium distribution coefficient. For linearly sorbing or even conservative tracers, such as tritiated water considered here, the transport Equation (7) is simplified to

$$
\frac{\partial C}{\partial t}=\frac{D_{\mathrm{e}}}{\alpha}\left(\frac{\partial^{2} C}{\partial r^{2}}+\frac{1}{r} \frac{\partial C}{\partial r}\right) .
$$

In this equation $D_{\mathrm{e}}\left(\mathrm{m}^{2} / \mathrm{s}\right)$ is the effective diffusion constant $\left(D_{\mathrm{e}}=\varepsilon D_{\mathrm{p}}\right)$ and $\alpha=\varepsilon+(1-\varepsilon) \rho K_{\mathrm{d}}(-)$ denotes the rock capacity factor and accounts for linear sorption processes. For conservative tracers, i.e. $K_{\mathrm{d}}=0, \alpha$ is reduced to the connected (transport) porosity of the OPA sample.

An appropriate initial condition is:

$$
C(r, t)=0 \quad r \in\left[r_{\mathrm{int}}, r_{\mathrm{ext}}\right], \quad t \leq 0,
$$

meaning that the clay sample is originally free of tracer. Suitable boundary conditions are as follows:

$$
\begin{aligned}
& C\left(r_{\text {int }}, t\right)=C_{0} \approx \text { const. }, \quad t>0, \\
& C\left(r_{\mathrm{ext}}, t\right) \approx 0, \quad t>0,
\end{aligned}
$$

where $\left(r_{\mathrm{ext}}-r_{\mathrm{int}}\right)(\mathrm{m})$ is the thickness of the cylindrical diffusion barrier. 
There is an analytical solution known for this type of diffusion problem. According to Equation (5.62) on page 84 of Crank (1975) or Equation (15) on page 207 of Carslaw and Jaeger (1959) the solution of the diffusion problem with constant boundary conditions is given by the following expression:

$$
\begin{aligned}
C(r, t)= & C_{0} \frac{\ln \left(r_{\mathrm{ext}} / r\right)}{\ln \left(r_{\mathrm{ext}} / r_{\mathrm{int}}\right)} \\
& +\pi C_{0} \sum_{n=1}^{\infty} \frac{J_{0}\left(r_{\mathrm{int}} \alpha_{n}\right) J_{0}\left(r_{\mathrm{ext}} \alpha_{n}\right) U_{0}\left(r \alpha_{n}\right)}{J_{0}^{2}\left(r_{\mathrm{int}} \alpha_{n}\right)-J_{0}^{2}\left(r_{\mathrm{ext}} \alpha_{n}\right)} \exp \left(-\frac{D_{\mathrm{e}} t}{\alpha} \alpha_{n}^{2}\right),
\end{aligned}
$$

with

$$
U_{0}\left(r \alpha_{n}\right)=J_{0}\left(r \alpha_{n}\right) Y_{0}\left(r_{\mathrm{ext}} \alpha_{n}\right)-J_{0}\left(r_{\mathrm{ext}} \alpha_{n}\right) Y_{0}\left(r \alpha_{n}\right),
$$

and where the $\alpha_{n}\left(\mathrm{~m}^{-1}\right)$ are the roots of $U_{0}\left(r_{\text {int }} \alpha_{n}\right)=0$.

Proceeding from Equations (12) one can get values for the diffusive flux $j(r, t)$ across the upper and the down-stream boundary. For the tracer flux $j\left(r_{\text {ext }}, t\right)$ across the boundary at the low-concentration side at $r=r_{\text {ext }}$ the following equation is obtained:

$$
\begin{aligned}
j\left(r_{\mathrm{ext}}, t\right) & =-\left.D_{\mathrm{e}} \frac{\partial C}{\partial r}\right|_{r=r_{\mathrm{ext}}} \\
& =\frac{C_{0} D_{\mathrm{e}}}{r_{\mathrm{ext}}}\left[\frac{1}{\ln \left(r_{\mathrm{ext}} / r_{\mathrm{int}}\right)}+2 \sum_{n=1}^{\infty} \frac{J_{0}\left(r_{\mathrm{int}} \alpha_{n}\right) J_{0}\left(r_{\mathrm{ext}} \alpha_{n}\right)}{J_{0}^{2}\left(r_{\mathrm{int}} \alpha_{n}\right)-J_{0}^{2}\left(r_{\mathrm{ext}} \alpha_{n}\right)} \exp \left(-\frac{D_{\mathrm{e}} t}{\alpha} \alpha_{n}^{2}\right)\right] .
\end{aligned}
$$

At short times there is a transient behaviour of the system while the porosity comes into contact with the diffusing solute and while the concentrations in the liquid and on the solid phase come into equilibrium. Later, a $1 / r$-concentration gradient (Figure 2) is established in the sample according to the leading term of Equation (13a), and the diffusion is in steady state with a constant flux, which is equal to

$$
j\left(r_{\mathrm{ext}}, t \rightarrow \infty\right)=\frac{C_{0} D_{\mathrm{e}}}{r_{\mathrm{ext}}} \frac{1}{\ln \left(r_{\mathrm{ext}} / r_{\mathrm{int}}\right)} .
$$

This latter quantity is independent of retarding mechanisms such as sorption. In the steady-state limit the following relationship for the fluxes holds:

$$
\frac{j\left(r_{\mathrm{int}}, t\right)}{j\left(r_{\mathrm{ext}}, t\right)}=\frac{r_{\mathrm{ext}}}{r_{\mathrm{int}}}
$$

hence, the fluxes $\left(\mathrm{Bq} \mathrm{m}^{-2} \mathrm{~s}^{-1}\right)$ across the boundaries are not equal to each other; however, the mass flow $\left(\mathrm{Bq} \mathrm{s}^{-1}\right)$ is conserved. 


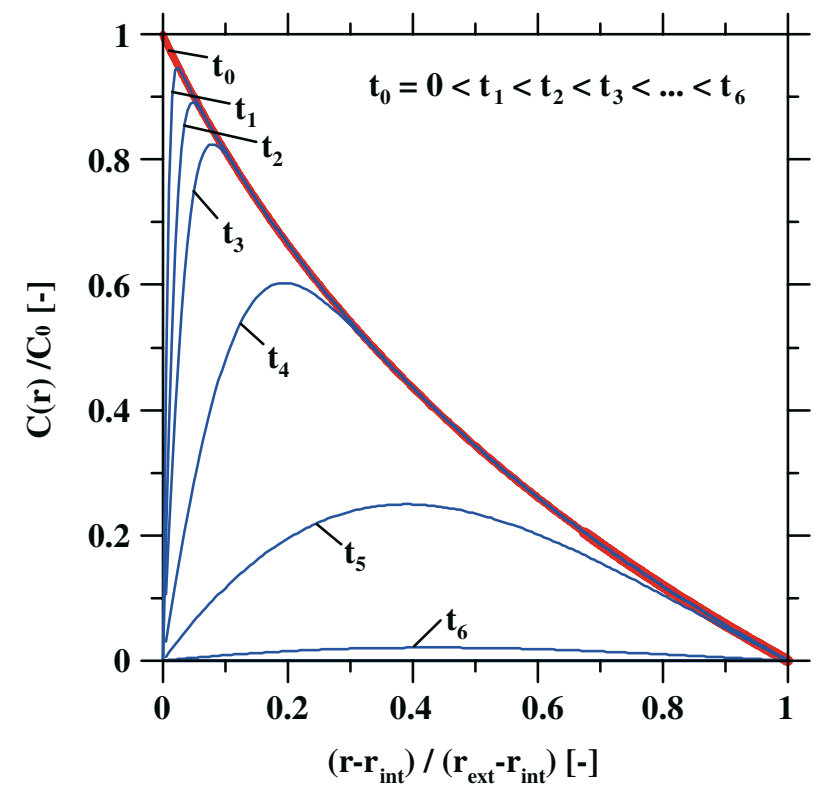

Figure 2. The logarithmic tracer concentration profile according to the leading term of Equation (12a) or Equation (15) after reaching the steady state in the throughdiffusion phase. In the out-diffusion phase, for times $t_{0}<t_{1}<t_{2}<\ldots$, the concentration profile decreases rapidly for conservative or only weakly sorbing tracers. In an early stage there are fast changes in the concentration gradient close to the inner boundary $r_{\text {int }}$ only.

By time-integration of the expression for the flux one obtains the time-dependent values for the total diffused mass across the down-stream boundary according to:

$$
\begin{aligned}
& m\left(r_{\mathrm{ext}}, t\right) \\
& =2 \pi r_{\mathrm{ext}} h \alpha \int_{0}^{t} j\left(r_{\mathrm{ext}}, t^{\prime}\right) \mathrm{d} t^{\prime} \\
& =2 \pi h C_{0} \alpha\left\{\frac{\left(\frac{D_{\mathrm{e}} t}{\alpha}-L\right)}{\ln \left(\frac{r_{\mathrm{ext}}}{r_{\mathrm{int}}}\right)}-2 \sum_{n=1}^{\infty} \frac{J_{0}\left(r_{\mathrm{int}} \alpha_{n}\right) J_{0}\left(r_{\mathrm{ext}} \alpha_{n}\right)}{J_{0}^{2}\left(r_{\mathrm{int}} \alpha_{n}\right)-J_{0}^{2}\left(r_{\mathrm{ext}} \alpha_{n}\right)} \frac{1}{\alpha_{n}^{2}} \exp \left(-\frac{D_{\mathrm{e}} t}{\alpha} \alpha_{n}^{2}\right)\right\},
\end{aligned}
$$

where $L\left(\mathrm{~m}^{2}\right)$ is a geometry factor

$$
L=\frac{\left(r_{\mathrm{int}}^{2}-r_{\mathrm{ext}}^{2}\right)+\left(r_{\mathrm{int}}^{2}+r_{\mathrm{ext}}^{2}\right) \ln \left(r_{\mathrm{ext}}-r_{\mathrm{int}}\right)}{4 \ln \left(r_{\mathrm{ext}}-r_{\mathrm{int}}\right)} .
$$

In this expression $2 \pi r_{\mathrm{ex}} h\left(\mathrm{~m}^{2}\right)$ is the geometrical surface area of the hollow cylinder at the down-stream (outer) boundary and $\alpha$ accounts for the 
porous medium and possible tracer uptake by sorption in terms of a linear isotherm formalism.

For the out-diffusion of radionuclides the same basic assumptions as for through-diffusion were applied. In order to model out-diffusion after a through-diffusion phase one has again to solve the 1D-diffusion Equation (8) with an initial tracer distribution according to the leading term of Equation (12a); hence

$$
C\left(r_{\text {int }}<r<r_{\text {ext }} ; t \leq 0\right)=C_{0} \frac{\ln \left(r_{\mathrm{ext}} / r\right)}{\ln \left(r_{\mathrm{ext}} / r_{\mathrm{int}}\right)} .
$$

The corresponding boundary conditions are as follows:

$$
C\left(r_{\mathrm{int}}, t>0\right)=C\left(r_{\mathrm{ext}}, t>0\right)=0 .
$$

Both the through- and the out-diffusion problems were solved with the help of the subroutine "MOLCH/DMOLCH" of the IMSL/MATHlibrary (IMSL, 1989). Although the inverse modelling was done purely numerically the Equations (12)-(15) were used for code verification purposes and a critical judgement of the modelling results. Furthermore, some of the mentioned equations cannot be found in textbooks such as, e.g., Crank (1998). With "MOLCH/DMOLCH" a system of coupled secondorder partial differential equations can be solved with an arbitrary initial condition and mixed von Neumann/Dirichlet boundary conditions using the method of lines. In this package cubic Hermite polynomials are used for the spatial approximation and Gear's method for stiff systems for the time integration is applied. The output of "MOLCH/DMOLCH" represents the time-history of, either $j\left(r_{\mathrm{ext}}, t\right)$, the flux across the outer boundary in the case of through-diffusion, or the fluxes at both boundaries, $r_{\text {int }}$ and $r_{\text {ext }} j\left(r_{\text {int }}, t\right)$ and $j\left(r_{\text {ext }}, t\right)$ respectively, in the case of tracer out-diffusion. These data are numerically integrated with the help of the simple Simpson's rule according to the integral expression of Equation (15a) in order to obtain values for the total (through- or out-) diffused tracer mass.

\section{The Fit Procedure}

The measured flux data, $j\left(r_{\text {ext }}, t\right)$ and $j\left(r_{\text {int }}, t\right)$ respectively, were used as the basis for the regression algorithm with $\left(D_{\mathrm{e}}, K_{\mathrm{d}}\right)$ as regression parameters. (Note: There are indeed two independent fit parameters due to the fact that in the through-diffusion phase the steady-state gives a value for $D_{\mathrm{e}}$ and the transient phase allows adjustment of $K_{\mathrm{d}}$ independently.) In practice we have taken the parameters as determined, neglecting the fact that they do not have the same order of magnitude. To avoid this we could have used logarithms; however, we did not investigate the effect on the best-fit values of the 
parameters. Assuming that a particular model represents the most relevant physical processes quite well, for the fitting procedure the modified Levenberg-Marquardt method was used by minimising of the $\chi^{2}$-merit function with the help of the IMSL/STAT-library function "RNLIN/DRNLIN". The automatised fitting procedure yielded subsequent error estimates and correlation coefficients between the fit parameters. Further details about the fitting procedure can be found in the IMSL-Library (1989).

It was important to apply the fitting-procedure several $(20-50 \ldots)$ times using different starting values for the fit-parameters in order to ensure that the global minimum of the $\chi^{2}$-merit function had finally been located. (In principle, by analysing the contours of the $\chi^{2}$-merit function in the $\left(D_{\mathrm{e}}\right.$, $K_{\mathrm{d}}$ )-plane the global minimum could also be evaluated.)

\section{Inverse Modelling, Results and Discussion}

For the numerical work, some parameter values had to be fixed. The necessary data are specified in Table II. The porosity of the sample was fixed to a total value of $15 \%$ based on many individual measurements on samples from Benken (Figure 3, from Gimmi (2003) and Gimmi (private communication)). Hence, larger total values for $\varepsilon$ are considered to be inconsistent with those observations.

Due to diffusing tracer and possible uptake by the solid phase, the reservoir concentration $C_{0}$ is not constant during the experiment. Hence, $C_{0}$ slowly decreases with time as can be seen in Figure 4. For the analysis of

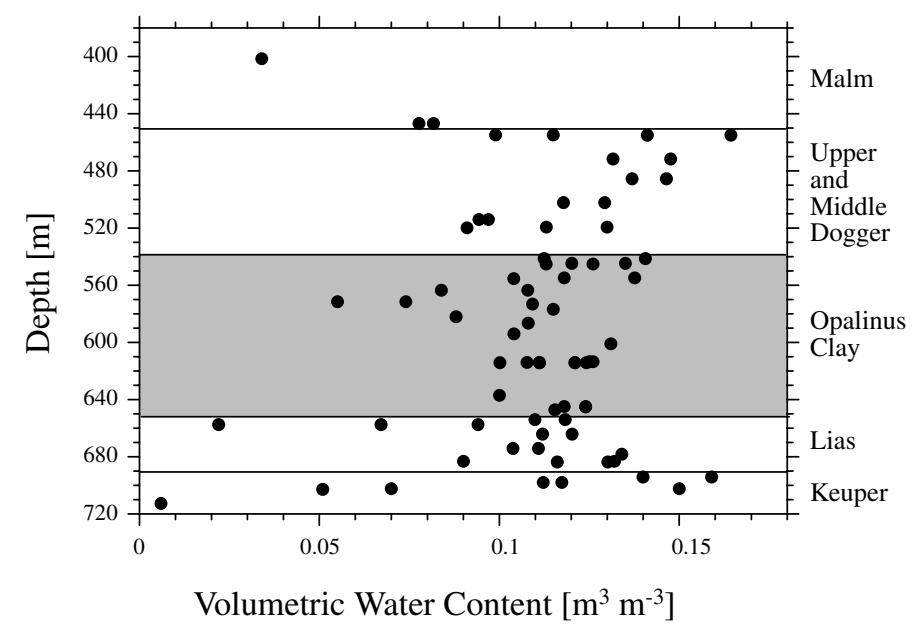

Figure 3. Measured porosity data from the Benken deep drilling for the OPA and the sedimentary layers above and below. As can be seen from the graph an upper

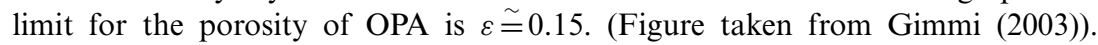




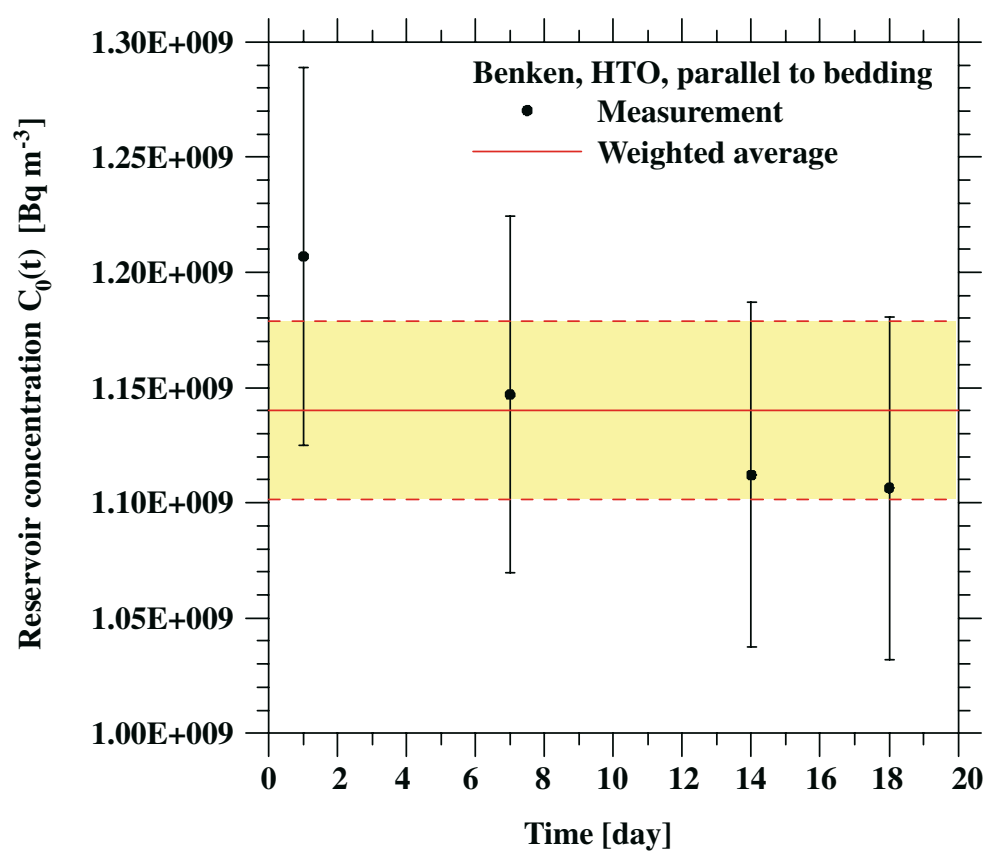

Figure 4. Measured tracer concentration in the reservoir compartment versus time. Also shown is the value of the weighted average (solid line) together with its $1 \sigma$-standard error which was used as (constant) boundary condition for the subsequent analysis of the through-diffusion experiment.

the experiments, however, we applied the (constant) weighted average of $C_{0}$ (see Table II).

In the frame of a 1D single porous medium diffusion/sorption model, the measured breakthrough data for $j\left(r_{\mathrm{ext}}, t\right)$ of the through-diffusion phase were fitted to the numerical solution of Equation (8) applying the procedure outlined in the previous subsection (Figure 5). The fit-parameters were $D_{\mathrm{e}}$ and $K_{\mathrm{d}}$. An excellent reproduction of the measurements could be achieved except for the first data point. As a crucial test of the diffusion/sorption model, the best-fit parameter values were then applied to blindly predict tracer out-diffusion across both boundaries at $r_{\text {int }}$ and $r_{\text {ext }}$. As can be seen in Figure 6, the model failed to accurately predict the data especially for times larger than 10 days. From the measurements of the outdiffusion phase a clear indication for the presence of a second transportrelevant porosity was obtained which could not be observed in the former through-diffusion phase. Hence, a model refinement was necessary. A further series of calculations was performed in the frame of a 1D dual porosity diffusion/sorption model accounting now for a second independent transport porosity. Hence, a linear superposition for the fluxes was made at the downstream boundary. Consequently, possible mixing of the tracer 


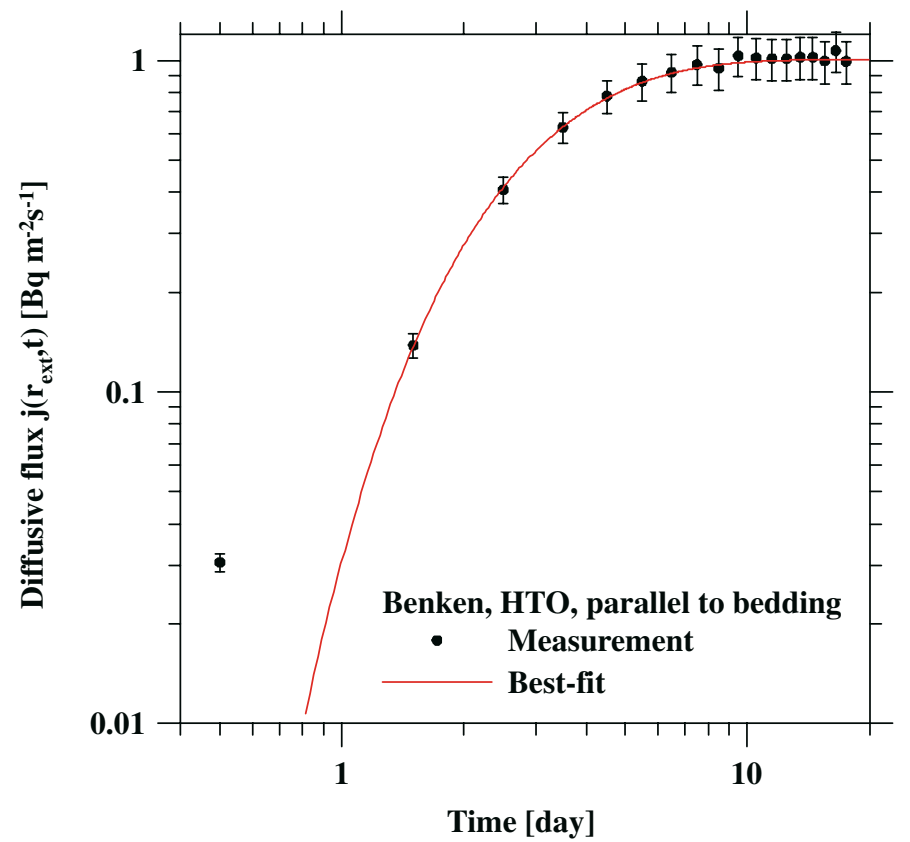

Figure 5. Measured and computed HTO breakthrough in the through-diffusion phase. The model considers only tracer transport in a single porosity medium. Except for the first data point there is excellent agreement between measurements and model. (This "outlier" is considered an indication that in reality there is a heterogeneous porosity distribution with a small amount of fast transport pathways whereas in our model only one type of porosity was considered.)

in the clay sample was entirely neglected and tracer transport occurs in our model in both types of porosity in parallel. In principle, there are now five adjustable parameters: for each of the porosities the effective diffusivity and the $K_{\mathrm{d}}$, as well as the weighing factor for both porosities. However, for reasons of simplicity and to reduce the number of fitting parameters we assumed that the sorption capacity should be the same for both porosities. For the sum of the porosities, i.e. for the total porosity, we assumed in addition again $15 \%$ as a constraint for the fit-procedure. Hence, there were finally four parameters which could be adjusted in a systematic way resulting in a unique solution of the inverse problem. As can be seen in Figures 7 and 8 with the refined model a satisfactory reproduction of the data for both diffusion phases could be achieved. The best-fit parameter values together with their $1 \sigma$-errors are compiled in Table IV. As can be seen, the value for the effective diffusivity is roughly an order of magnitude smaller for the second type of pores and the values for the porosity differ only by a factor of two. However, further information about the pore size distribution cannot be deduced since such a possible relationship between diffusivity and pore size distribution is unknown. Since the fitting procedure 


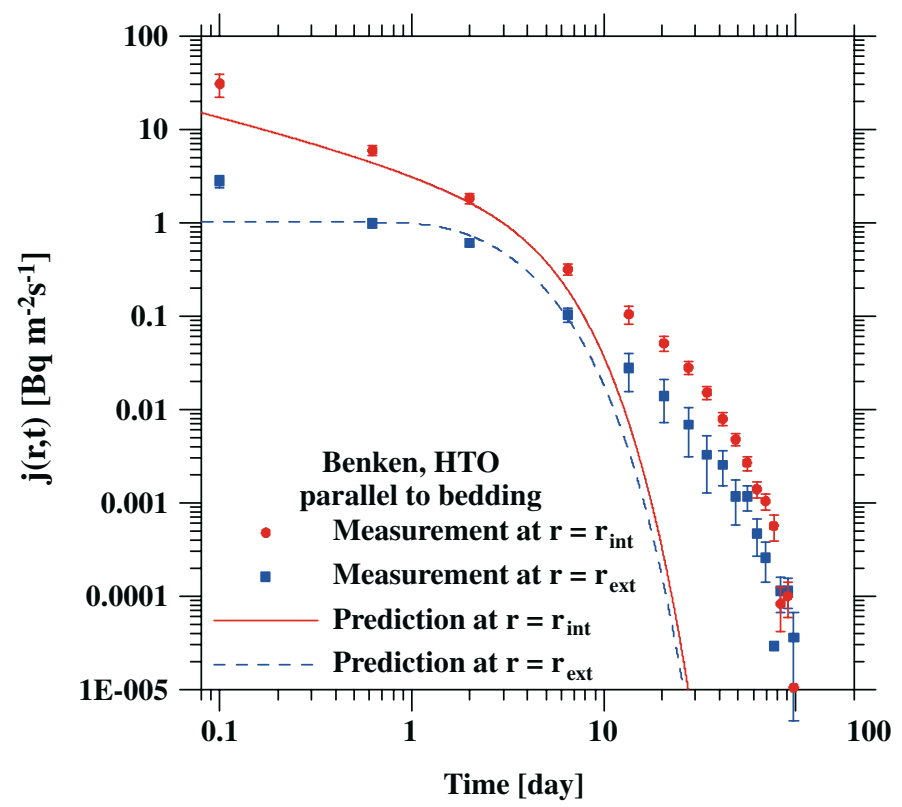

Figure 6. Blind-prediction of tracer out-diffusion together with the measurements. Shown are the values for the diffusive fluxes across the inner (subscript int) and the outer boundary (subscript ext) of the hollow cylindrical clay sample. As can be seen, the model fails to predict the data for later times (for $t>10$ days), although it is based on the best-fit of the former through-diffusion phase.

resulted in values for the rock capacity factor larger than 0.15 (the value for the maximum total porosity) a non-zero $K_{\mathrm{d}}$-value - in the order of $K_{\mathrm{d}} \approx 10^{-5} \mathrm{~m}^{3} \mathrm{~kg}^{-1}$ - had to be accepted. Unfortunately, the extracted $K_{\mathrm{d}}$ value for sorption cannot be compared with $R_{\mathrm{d}}$ values from static batchsorption experiments due to its low value, which is roughly an order below the detection limit for batch sorption experiments. (Note that $R_{\mathrm{d}}$ determined in batch sorption experiments is defined as the ratio $S / C$ where $S$ is the amount of tracer absorbed onto the solid phase per unit mass of solid phase and $C$ is the amount of tracer in solution per unit volume of solution. However, $K_{\mathrm{d}}$ is defined as $\mathrm{d} S / \mathrm{d} C$ and is therefore a modeldependent quantity. In the case where sorption is described by a linearisotherm formalism according to $S=K_{\mathrm{d}} C, R_{\mathrm{d}} \equiv K_{\mathrm{d}}$ holds.) The observed bi-modal tracer breakthrough could - in principle - be caused by various processes such as spatially variable (i.e. mineral) dependent sorption, anisotropic diffusion etc. As already mentioned we applied one single $K_{\mathrm{d}^{-}}$ value for both types of pores only. Presumably there will be (at least) two different values for $K_{\mathrm{d}}$, however presently no information about that issue is available. Moreover, in our study we focussed on the effects of heterogeneities of the transport-relevant pore space only and based on the following 


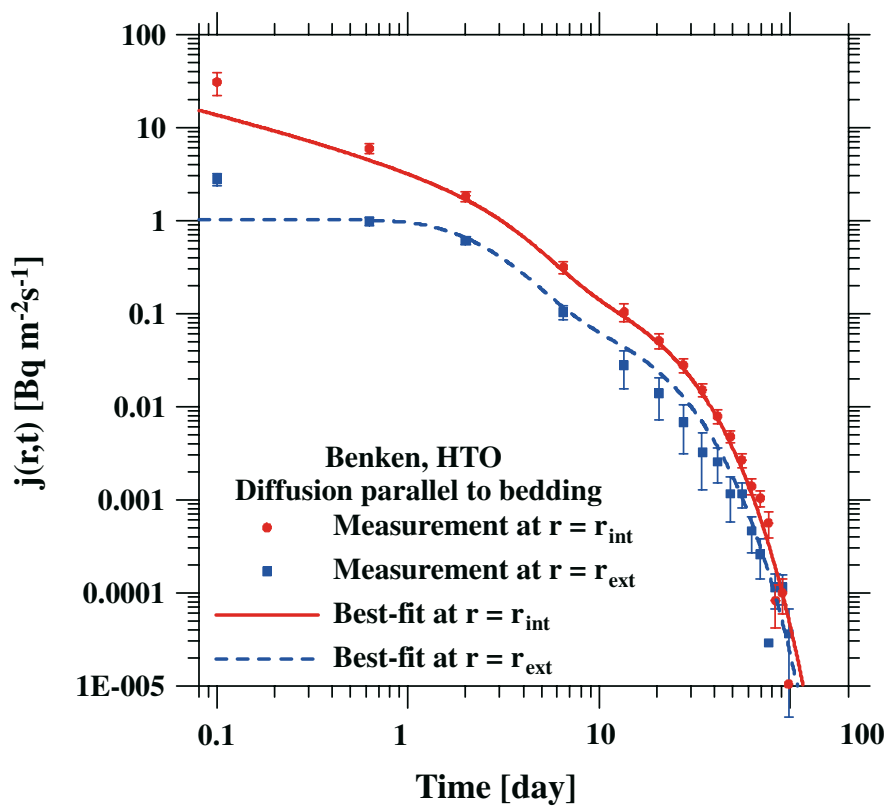

Figure 7. Best-fit of the out-diffusion data in the frame of a $1 \mathrm{D}$ dual-porosity model. Shown are the measured and the computed data for both boundaries. (For simplicity reason only, the individual contributions of the two different porosities to total flux are not drawn. However, their values are compiled in Table IV. The small differences between measurements and model at the outer boundary at $r_{\mathrm{ext}}$ are due to the fact that the experimental tracer flux through the second type of pores was not yet in steady state when starting tracer out-diffusion (see also Figure 8). For the modelling, however, a steady-state initial condition was assumed for both types of pores and, hence, the computed flux values at the outer boundary are slightly larger than the measurements. Note: the very first measurements are influenced by uncontrolled artefacts due to changing the boundary conditions after the throughdiffusion phase.

picture of OPA. Regarding the details of the OPA concerning texture on the micrometer scale (Figure 9), there are in principle three different types of water present: (a) interlayer water - the water between the TOT units of smectite present in OPA; (b) double layer water associated with the external surface of the clay stacks; and (c) free water which denotes the interconnected thin films on the outside of the clay stacks (but not part of the electrical double layer) and other mineral grains (Nagra, 2002a). Based on our measurements and the subsequent analysis, however, it is not possible to determine which parts of the total pore space account for the observed bi-modal tracer breakthrough in the out-diffusion phase. For that, more information from through- and out-diffusion experiments, from sorption studies applying all kind of tracers - neutral, anionic and cationic and spectroscopic investigations on the micrometer (e.g. $\mu$-XAS) or even 


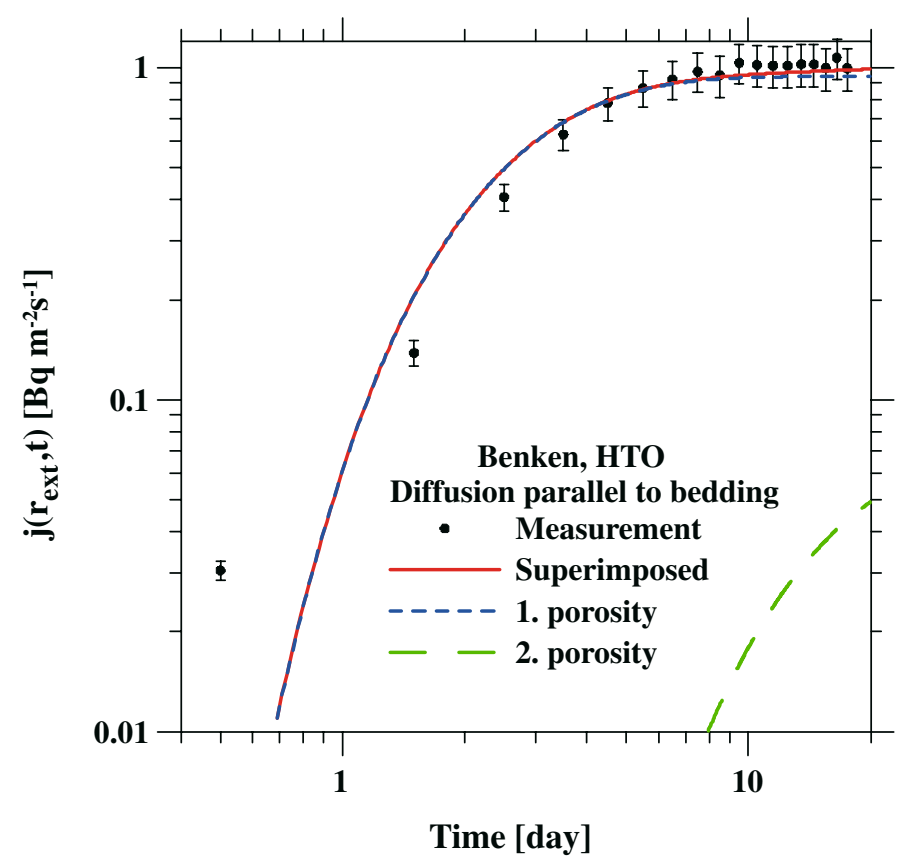

Figure 8. Best-fit of the through-diffusion data using a 1D dual-porosity model. The best-fit parameter values are the same as for modelling the subsequent out-diffusion phase. The second porosity accounts only for late breakthrough times to total tracer flux and hence, those small effects can hardly be recognized in the breakthrough data. Note: After 20 days, a steady-state condition was reached only for the first type of porosity.

nanometer scale (e.g. neutron spectroscopy) on clays is necessary. These investigations are partially already going on or are planned for the near future. Their results will finally provide the scientific basis for an increased understanding regarding the mobility of highly dissolved solutes in argillaceous rocks.

Finally, the amount of tracer which has diffused is obtained by time integration of the tracer flux (see Equations (15)). The calculation, in agreement with the observations, indicated that roughly $30 \%$ of total tracer diffused through the second porosity. Such a result is encouraging regarding performance assessments for repositories for radioactive waste hosted by OPA, since the second porosity is characterised by a decreased mobility of the tracer. Hence, accounting for a second porosity in future performance assessments will not only reduce conservatisms but the procedure has - in principle - the potential to reduce further total radiological doses, especially for some safety-relevant radionuclides, provided the required data will be available and the necessary system understanding can be developed in the meantime. 
Table IV. Best-fit parameter values together with their one standard deviation errors for HTO as tracer and OPA from the Benken deep drilling as sample applying a confining pressure of $15 \mathrm{MPa}$. ( $D_{\mathrm{a}}$ is the apparent diffusion coefficient: $D_{\mathrm{a}}=D_{\mathrm{e}} / \alpha$.) The four freely adjustable parameters were $\varepsilon, D_{\mathrm{e}}$ and $K_{\mathrm{d}}$; for the porosities the following constraint holds: $\sum \varepsilon=0.15$. All the other parameter values are deduced from the four fit parameters and are mentioned for illustration purposes only

\begin{tabular}{llc}
\hline Parameter & Unit & Value \\
\hline 1. Porosity & & \\
$\varepsilon$ & - & $0.10 \pm 0.01$ \\
$\alpha$ & - & $0.117 \pm 0.010$ \\
$D_{\mathrm{e}} \cdot 10^{11}$ & $\mathrm{~m}^{2} \mathrm{~s}^{-1}$ & $2.8 \pm 0.3$ \\
$D_{\mathrm{p}} \cdot 10^{11}$ & $\mathrm{~m}^{2} \mathrm{~s}^{-1}$ & $28 \pm 5$ \\
$D_{\mathrm{a}} \cdot 10^{11}$ & $\mathrm{~m}^{2} \mathrm{~s}^{-1}$ & $24 \pm 4$ \\
2. Porosity & & \\
$\varepsilon$ & & $0.05 \pm 0.01$ \\
$\alpha$ & - & $0.058 \pm 0.010$ \\
$D_{\mathrm{e}} \cdot 10^{11}$ & - & $0.22 \pm 0.02$ \\
$D_{\mathrm{p}} \cdot 10^{11}$ & $\mathrm{~m}^{2} \mathrm{~s}^{-1}$ & $4.4 \pm 1.0$ \\
$D_{\mathrm{a}} \cdot 10^{11}$ & $\mathrm{~m}^{2} \mathrm{~s}^{-1}$ & $3.8 \pm 0.8$ \\
\hline$K_{\mathrm{d}} \cdot 10^{5}$ & $\mathrm{~m}^{2} \mathrm{~s}^{-1}$ & $1.1 \pm 0.3$ \\
\hline
\end{tabular}

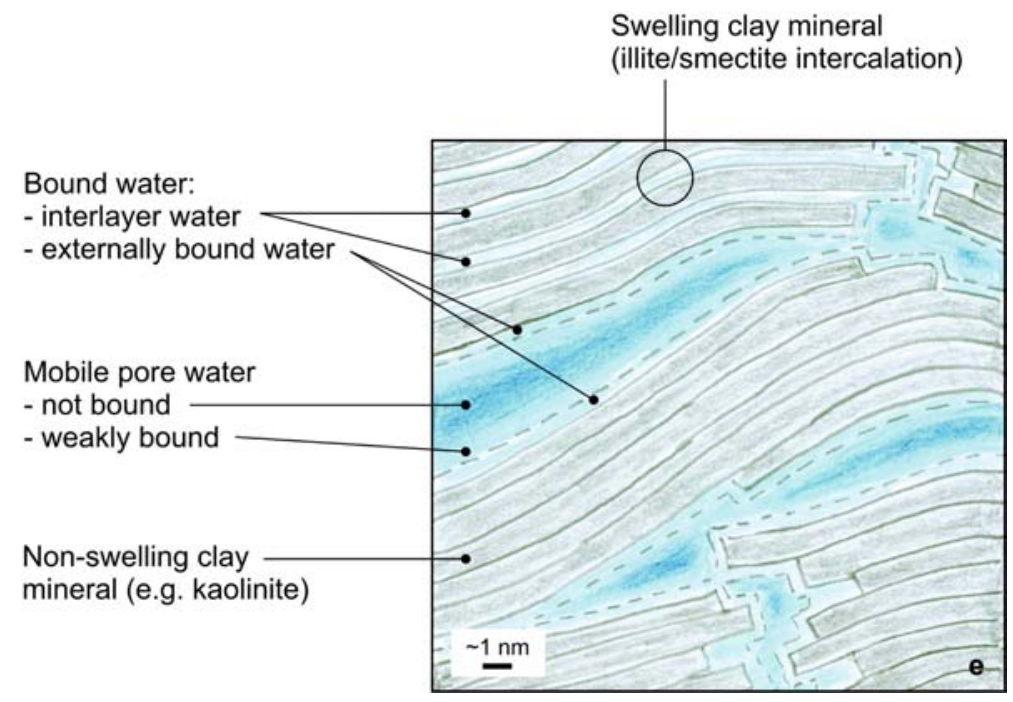

Figure 9. Simplified sketch of the OPA structure on the micrometer scale from (Nagra, 2002a, p. 82, Figure 4.2-13). The pore water-filled void space is composed of the "interlayer" and a complex 3D pore-network with "double layer water" and "free water" surrounding the clay platelets and the mineral grains. 


\section{Conclusions}

We performed through- and out-diffusion experiments with tritiated water in an OPA sample from a borehole core from the deep-drilling in Benken, Northern Switzerland. The experiments were performed parallel to the fabric of a hollow OPA cylinder and the modelling was done in cylindrical coordinates according to the experimental set-up. The experiments consisted of two different phases: (1) a through-diffusion phase until near steady state with regard to the diffusive tracer flux across the outer down-stream boundary; and (2) an out-diffusion phase where the sample, loaded with tracer from the previous through-diffusion phase, was leached.

This is a novel method yielding independent information on both boundaries concerning the diffusive tracer flux; it also provides information about the steady-state tracer distribution in the sample of the preceding through-diffusion phase, hence, on the homogeneity regarding transport properties of the sample. By inverse modelling both, tracer through- and out-diffusion, values for the effective diffusion coefficient and further information concerning sorption capacity and sample homogeneity could be deduced.

With the help of a simple 1D-diffusion/sorption model, the tracer through-diffusion data could be reproduced with high precision. Hence, the breakthrough data showed no irregularities. However, the temporal evolution of the diffusive tracer flux during the out-diffusion phase showed a more complex behaviour and the model failed to blindly predict tracer out-diffusion. Consequently, the model had to be refined by accounting of a second independent transport porosity. In the frame of the 1D dual porosity diffusion/sorption model (having now four instead of only two freely adjustable parameters) we could successfully reproduce both the breakthrough data from the through- and out-diffusion phase. The extracted best-fit parameter values for the effective diffusion coefficient are in the order of $3 \times 10^{-11} \mathrm{~m}^{2} / \mathrm{s}$ for the first (fast) type of porosity and roughly an order of magnitude smaller for the second porosity. In line with independent measurements concerning porosity we applied a maximum value for the total porosity of 0.15 as a constraint for the analysis. The analysis yielded values for the porosities of $10 \%$ and $5 \%$ for the first and second type of void space, respectively. However, due to the constraint we had to accept a small, but non-zero $K_{\mathrm{d}}$-value for tritium sorption in the order of $10^{-5} \mathrm{~m}^{3} / \mathrm{kg}$, since the values for the rock capacity factors were slightly larger than those of the porosities. Unfortunately, we are presently not in a position to further comment on these values, because independent batch sorption experiments cannot be performed due to the low sorption capacity for tritiated water. In order to shed light on the nature of the two different types of transport-relevant porosities, we will perform detailed 
mineralogical investigations especially with regard to the finer details of the fabrics of the OPA samples. In addition, we will continue with this type of combined diffusion experiments using cationic and anionic tracers. Spectroscopic methods such as $\mu$-XAS and neutron-spectroscopy will also contribute to increase our understanding of the transport of solutes through clays on a fundamental level.

Finally, roughly one third of the total amount of tracer migrated through the second porosity with increased retardation. Such a behaviour might be of interest for future performance assessments for repositories for radioactive waste.

\section{Acknowledgements}

This work was partially funded by the Swiss National Cooperative for the Disposal of Radioactive Waste (Nagra). Dr. L. Johnson (Nagra) is acknowledged for the comments on the manuscript.

\section{References}

Andra, Dossier: 2001, Argile. Progress report on feasibility studies \& research into deep geological disposal of high-level, long-lived waste. Synthesis report. Andra, Paris, France.

Bourke, P. J., Jefferies, N. L., Lever, D. A. and Lineham, T. R.: 1993, Mass Tranfer mechanisms in compacted clays, in: D.A.C. Manning, P.L. Hall and C.R. Hughes (Eds), Geochemistry of Clay-Pore Fluid Interactions, Chapman \& Hall, London.

Carslaw, H. S. and Jaeger, J. C.: 1959, Conduction of Heat in Solids. Oxford at the Carendon Press, United Kingdom.

Crank, J.: 1998, The Mathematics of Diffusion. Oxford Science Publications, Clarendon Press, Oxford, United Kingdom, Second edition.

Gimmi, T.: 2003, Porosity, pore structure, and energy state of pore water of Opalinus clay from Benken. Nagra, Wettingen, Switzerland. Unpublished report.

Henrion, P. N., Put, M. J. and Van Gompel, M.: 1991, The influence of compaction on the diffusion of non-sorbed species in Boom Clay. Radioactive Waste Manage. Nucl Fuel Cycle 16, 1-14.

IMSL-Library: 1989, Edition 1.1.

Nagra: 2001, Sondierbohrung Benken: Untersuchungsbericht. Nagra Technical Report NTB 00-01, Nagra, Wettingen, Switzerland.

Nagra: 2002a, Project Opalinus Clay: Safety Report - Demonstration of disposal feasibility for spent fuel, vitrified high-level waste and long-lived intermediate-level waste (Entsorgungsnachweis). Nagra Technical Report NTB-02-05, Nagra, Wettingen, Switzerland.

Nagra: 2002b, Projekt Opalinuston: Synthese der geowissenschaftlichen Untersuchungsergebnisse - Entsorgungsnachweis für abgebrannte Brennelemente, verglaste hochaktive sowie langlebige mittelaktive Abfälle. Nagra Technischer Bericht NTB 02-03, Nagra, Wettingen, Schweiz (in German).

Ondraf/Niras: 2001, Technical overview of the SAFIR 2 report. Technical Report NIROND 2001-05 E, Brussels, Belgium.

Pearson, F. J.: 2000, Nagra unpublished internal report, Nagra, Wettingen, Switzerland. 
Put, M. J. and De Canniere P.: 1994, Migration behaviour of ${ }^{14} \mathrm{C}$ labelled bicarbonate, HTO and ${ }^{131} \mathrm{I}$ in boom clay. Radiochim. Acta 66/67, 385-388.

Van Loon, L. R. and Soler, J. M.: 2004, Diffusion of $\mathrm{HTO},{ }^{36} \mathrm{Cl}-,{ }^{125} \mathrm{I}^{-}$and ${ }^{22} \mathrm{Na}^{+}$in Opalinus Clay: Effect of Confining Pressure, Sample Orientation, Sample Depth and Temperature. PSI Bericht 04-03, Paul Scherrer Institut, Villigen, Switzerland.

Van Loon, L. R., Soler, J. M. and Bradbury, M. H.: 2003a, Diffusion of HTO, ${ }^{36} \mathrm{Cl}^{-}$ and ${ }^{125} \mathrm{I}^{-}$in Opalinus clay samples from Mont Terri: effect of confining pressure. J. Contam. Hydrol. 61, 73-83.

Van Loon, L. R., Soler, J. M., Jakob, A. and Bradbury, M. H.: 2003b, Effect of confining pressure on the diffusion of $\mathrm{HTO},{ }^{36} \mathrm{Cl}^{-}$and ${ }^{125} \mathrm{I}^{-}$in a layered Argillaceous Rock (Opalinus clay): diffusion perpendicular to the fabric. Appl. Geochem. 18, 1653-1662.

Van Loon, L. R., Soler, J., Müller, W. and Bradbury, M. H.: 2004, Anisotropic diffusion in layered Argillaceous Rocks: a case study with Opalinus clay. Environ. Sci. Technol. 38, 5721-5728. 